\title{
CRINOIDEA DA FORMAÇÃO MAECURU (DEVONIANO DA BACIA DO AMAZONAS), ESTADO DO PARÁ, BRASIL
}

\author{
SANDRO MARCELO SCHEFFLER, ANTONIO CARLOS SEQUEIRA FERNANDES \& \\ VERA MARIA MEDINA DA FONSECA \\ Departamento de Geologia e Paleontologia, Museu Nacional,UFRJ, Quinta da Boa Vista, São Cristóvão, 20940-040, \\ Rio de Janeiro, RJ, Brasil.schefflersm@yahoo.com.br, fernande@acd.ufrj.br,vmedina@acd.ufrj.br
}

\begin{abstract}
RESUMO - Na Formação Maecuru (Devoniano Médio da bacia do Amazonas) é muito comum a ocorrência de partes desarticuladas de crinóides juntamente com exemplares de braquiópodes e moluscos. Em seis amostras de arenito da citada formação, contendo centenas de partes dissociadas de pedúnculos e cálices, foram identificadas as seguintes espécies de crinóides: Laudonomphalus regularis Moore \& Jeffords, 1968 e L. ornatus Moore \& Jeffords, 1968; Exaesiodiscus aff. minutus Moore \& Jeffords, 1968; e Monstrocrinus securifer Schmidt, 1941. A ocorrência de Monstrocrinus é rara no Devoniano e considerada até o presente restrita ao Devoniano Inferior (topo do Emsiano) da Alemanha, Espanha e Argélia. Sua ocorrência na Formação Maecuru reveste-se de especial importância por serem idênticos a espécie-tipo do gênero, M. securifer Schmidt 1941, do Maciço Xistoso Renano (Alemanha), sugerindo relação entre as faunas das duas regiões. É o primeiro registro do gênero na América do Sul, e a primeira ocorrência no Devoniano Médio (Eifeliano). A presença de M. securifer na Formação Maecuru fornece subsídios para interpretações paleobiogeográficas relacionadas ao padrão de distribuição e prováveis rotas de migração de invertebrados bentônicos no Gondwana Norte durante o Devoniano.
\end{abstract}

Palavras-chave: Crinoidea, Rhodocrinitidae, ø Flucticharacidae, ø Exaesiodiscidae, Devoniano, bacia do Amazonas.

ABSTRACT - CRINOIDEA OF MAECURU FORMATION (DEVONIAN OF AMAZON BASIN), PARÁ STATE, BRASIL. The desarticulated parts of crinoids are very common in Maecuru Formation (Middle Devonian, Amazonas Basin), occurring with brachiopods and mollusks. In six sandstone samples from this formation, including hundreds of detached calix and stem parts it was identified the following species: Laudonomphalus regularis Moore \& Jeffords, L. ornatus Moore \& Jeffords, Exasiodiscus aff. minutus Moore \& Jeffords, and Monstrocrinus securifer Schimdt. The Monstrocrinus occurrence is rare in the Middle Devonian, being registered until now in Germany, Spain and Algeria Lower Devonian (upper Emsian). This occurrence in Maecuru Formation is specially important, because the specimens are identical to the type species ofte genus, M. securifer Schmidt, from the Rhenish slates Massif. This suggests relation between the faunas of these regions. This is the first Monstrocrinus record in South America and in Middle Devonian (Eifelian). These facts give subsidies to paleogeographic interpretations regarding to invertebrate distribution patterns and suggest probable migration routes in North of Gondwana Devonian.

Key words: Crinoidea, Rhodocrinitidae, ø Flucticharacidae, ø Exaesiodiscidae, Devonian, Amazon Basin.

\section{INTRODUÇÃO}

Os crinóides são relativamente abundantes em unidades litoestratigráficas devonianas brasileiras. Entretanto, existem poucos estudos detalhados, sendo que a maioria das informações se limita apenas a citar ocorrências nas bacias do Amazonas (Formação Maecuru), Parnaíba (Formação Cabeças) e Paraná (Formação Ponta Grossa).

Os primeiros registros de crinóides na Formação Maecuru devem-se a Katzer $(1903,1933)$, que agrupou placas colunais isoladas, sob o gênero Ctenocrinus Bronn, 1840. Mais tarde, Moore \& Jeffords (1968) demonstraram que os segmentos isolados das colunas não permitiam uma classificação desses organismos dentro da sistemática formal do grupo, fato que os levou a propor uma parataxonomia. Com base nessa parataxonomia, Ferreira \& Fernandes (1985) referiram placas colunais dissociadas e segmentos pluricolunais de crinóides da Formação Maecuru, coletados pela Comissão Geológica do Império, aos gêneros Laudonomphalus Moore \& Jeffords, 1968 e Exaesiodiscus Moore \& Jeffords, 1968. Ferreira \& 
Fernandes (1989) registraram a presença de espinhos isolados e placas calicinais de grandes dimensões nas mesmas amostras, atribuindo-os ao gênero Monstrocrinus Schmidt, 1941 e, posteriormente, foram referidos à espécie $M$. securifer Schmidt, 1941 (Fernandes et al., 2000).

O objetivo do presente trabalho é descrever, pela primeira vez, as partes dissociadas dos crinóides presentes nos arenitos da Formação Maecuru, revisando sua classificação e avaliando o significado paleogeográfico da ocorrência de Monstrocrinus na bacia do Amazonas.

\section{CONTEXTO GEOLÓGICO}

Os arenitos fossilíferos onde ocorrem os crinóides são procedentes de afloramentos situados na faixa de estratos paleozóicos que ao norte se encontra paralela ao curso do rio Amazonas, no Estado do Pará (Figura 1). Esses sedimentitos constituem a parte superior da Formação Maecuru e, com a Formação Ererê, formam o registro do Devoniano Médio da bacia do Amazonas. A Formação Maecuru é constituída por camadas de arenitos e pelitos flúvio-deltaicos a neríticos. A predominância de pelitos geralmente caracteriza a subunidade inferior da formação, o Membro Jatapu, enquanto arenitos de granulometria fina a grossa predominam, sobretudo, na subunidade superior, o Membro Lontra. Localmente, no flanco norte e em subsuperfície, os sedimentitos mais superiores do Membro Lontra são tempestitos gerados em ambiente marinho raso, constituídos por arenitos com estratificação cruzada hummocky, com pouquíssimas intercalações pelíticas e contêm a fauna de invertebrados marinhos devonianos da Formação Maecuru. Juntamente com os crinóides, esses arenitos exibem restos de trilobitas, moluscos, briozoários, corais, conulárias e tentaculítidas (Melo, 1988; Cunha et al., 1994).

Datações palinológicas do Membro Lontra, consideramno de idade emsiana a eifeliana (Loboziac \& Melo, 2000, 2002; Figura 2). As camadas portadoras de macrofósseis conchíferos marinhos restringem-se à sua parte mais superior.

\section{LOCALIZAÇÃO GEOGRÁFICA}

Todas as amostras estudadas foram coletadas pela antiga Comissão Geológica do Império do Brasil em 1876, com exceção daquelas registradas como MN 7418-I, MN 8196-I a,b e MN 8197-I que foram coletadas pela Expedição Orville Adelbert Derby, patrocinada pela Petrobras, em 1986.

As amostras da Comissão Geológica do Império foram coletadas no rio Maecuru, a montante da cachoeira Teuapixuna, no Estado do Pará (Derby, 1878). As amostras da Expedição Orville Adelbert Derby foram coletadas na margem direita do mesmo rio, cerca de $400 \mathrm{~m}$ a montante da cachoeira Teuapixuna (ou Alagação).

\section{MATERIAL E MÉTODOS}

O material estudado, constituído por lajes de arenito de tamanho pequeno a médio contendo centenas de placas de crinóides, encontra-se depositado nas coleções dos setores

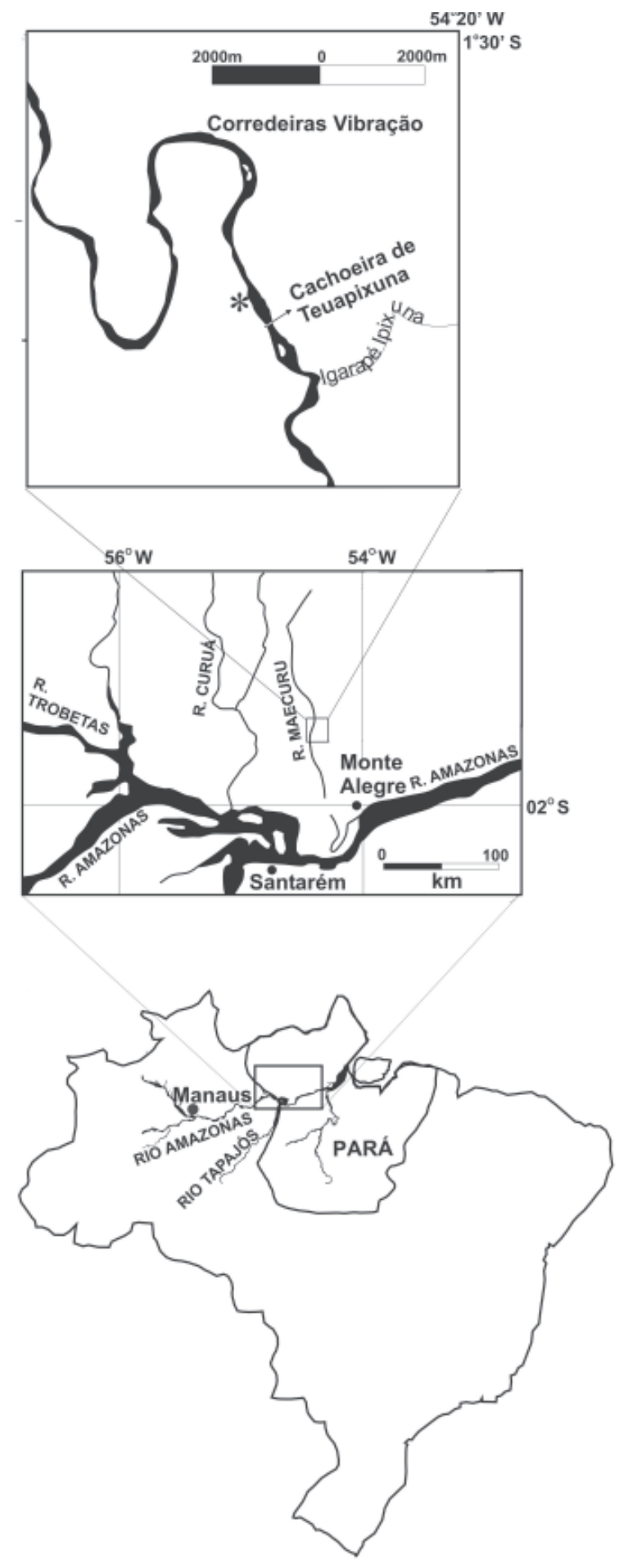

Figura 1. Mapa de localização com o ponto de coleta do material estudado (modificado de Machado et al., 1996).

Figure 1. Location map of the collecting site (modified from Machado et al., 1996).

de petrografia (MN 5164 e MN 5173a, b, c) e de paleoinvertebrados (MN 3412-I, MN 6133-I, MN 7418-I, MN 7581-I a MN 7587-I, MN 8196-Ia, b, e MN 8197-I) do Departamento de Geologia e Paleontologia do Museu Nacional da Universidade Federal do Rio de Janeiro.

Os restos de crinóides presentes na Formação Maecuru correspondem a três tipos básicos: (i) placas calicinais 


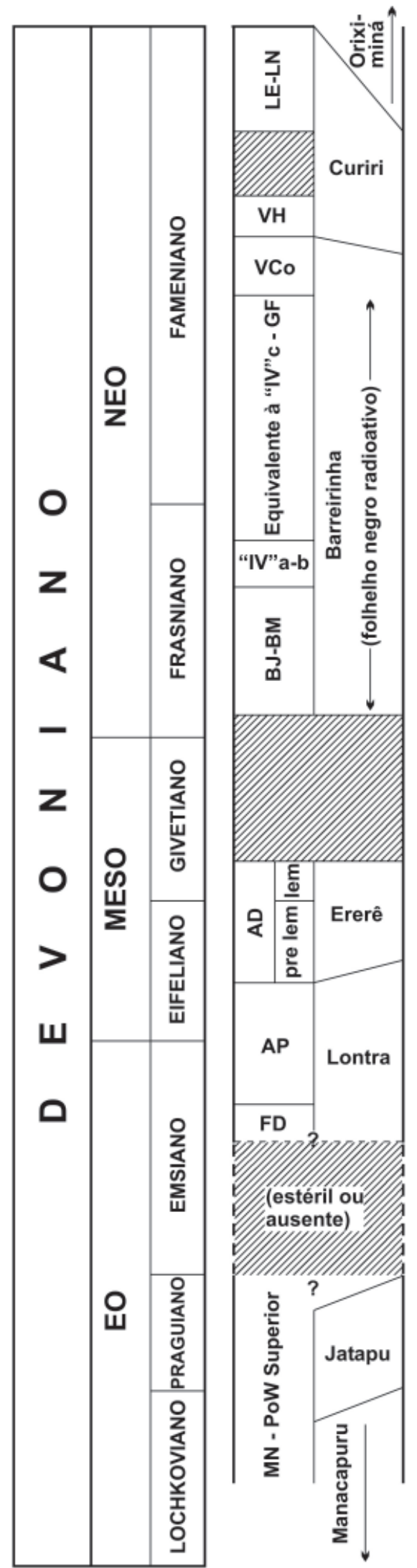

Figura 2. Biozonas de miósporos do Devoniano da bacia do Amazonas e correlação com as unidades litoestratigráficas regionais (modificado de Loboziak \& Melo, 2002).

Figure 2. Miospore biozones for the Devonian of the Amazon Basin and their correlation with regional lithostratigraphic units (modified from Loboziac \& Melo, 2002). isoladas; (ii) colunais isoladas (também denominadas artículos ou entrocas); e (iii) pluricolunais (segmentos da coluna com mais de duas colunais articuladas).

Para a identificação das colunais isoladas e pluricolunais dos gêneros Laudonomphalus e Exaesiodiscus foi utilizada a classificação parassistemática proposta por Moore \& Jeffords (1968) e, para as placas calicinais do gênero Monstrocrinus, a sistemática adotada por Le Menn (1990). A terminologia utilizada para a descrição das colunais isoladas e pluricolunais foi baseada em Moore et al. (1968).

As espécies baseadas exclusivamente na parassistemática são precedidas pelo símbolo "ø", o qual é utilizado em trabalhos de descrição de material fragmentário de crinóides (Le Menn, 1987b; Le Menn, 1988) e são indicadores práticos de táxons baseados em colunais e pluricolunais (Donovan, 2001).

Os símbolos empregados para as medidas e índices das colunais e pluricolunais são aqueles definidos em (Moore et al., 1968), a saber: A, aréola (areola); Ai, índice areolar (areolar index); F, faceta articular (articulum, articular facet); Fi, índice da faceta articular (articular facetal index); KD nodal, diâmetro da colunal nodal (columnal nodal diameter); KH nodal, altura da colunal nodal (columnal nodal height); KHi nodal, índice da altura da colunal nodal (columnal nodal height index); L, lúmen (lumen); Li, índice luminal (luminal index); $\mathrm{Ni}$, índice nodal (nodal index); NT, noditaxe (noditaxis); P, perilúmen (perilumen); $\mathrm{Pi}$, índice periluminal (periluminal index).

\section{PALEONTOLOGIA SISTEMÁTICA}

Classe CRINOIDEA Miller, 1821

Subclasse CAMERATA Wachsmuth \& Springer, 1885

Ordem DIPLOBATHRIDA Moore \& Laudon, 1943

Subordem EUDIPLOBATHRINA Ubaghs, 1953

Superfamília RHODOCRINITACEA Roemer, 1855

Família RHODOCRINITIDAE Roemer, 1855 Monstrocrinus Schmidt, 1941

Espécie-tipo. Monstrocrinus securifer Schmidt, 1941

Monstrocrinus securifer Schmidt, 1941 (Figura 3)

Material. MN 5164, MN 5173a3, MN 5173a4, MN 5173a5, MN5173b7, MN5173c6, MN 6133-Ia e MN 7418-Ia.

Descrição. Placas calicinais com dois tipos de expansões: uma larga em forma de machado (Figura 3A-D), a maior e mais completa (MN 5164) medindo aproximadamente $9,57 \mathrm{~mm}$ de largura na base e $46,45 \mathrm{~mm}$ no topo e cerca de $37,55 \mathrm{~mm}$ de altura, e outra em forma de espinhos longos e estreitos (Figura 3E) de base alargada e medidas variáveis, com o maior exemplar (MN 5173a5) medindo aproximadamente 8,34 $\mathrm{mm}$ de largura na base e pelo menos 20,88 mm de comprimento. Ambas as formas têm como característica comum a base côncava.

Discussão. Segundo Le Menn (1990), são conhecidas quatro espécies de Monstrocrinus, todas do Emsiano: M. securifer Schmidt,1941 e M. granosus Schmidt,1941, do maciço Xistoso Renano (Alemanha); M. aliformis Le Menn,1990, da região de Aragão (Espanha) e Monstrocrinus sp. dos Montes de Ougarta (Argélia). Entre os restos de crinóides da Formação Maecuru 

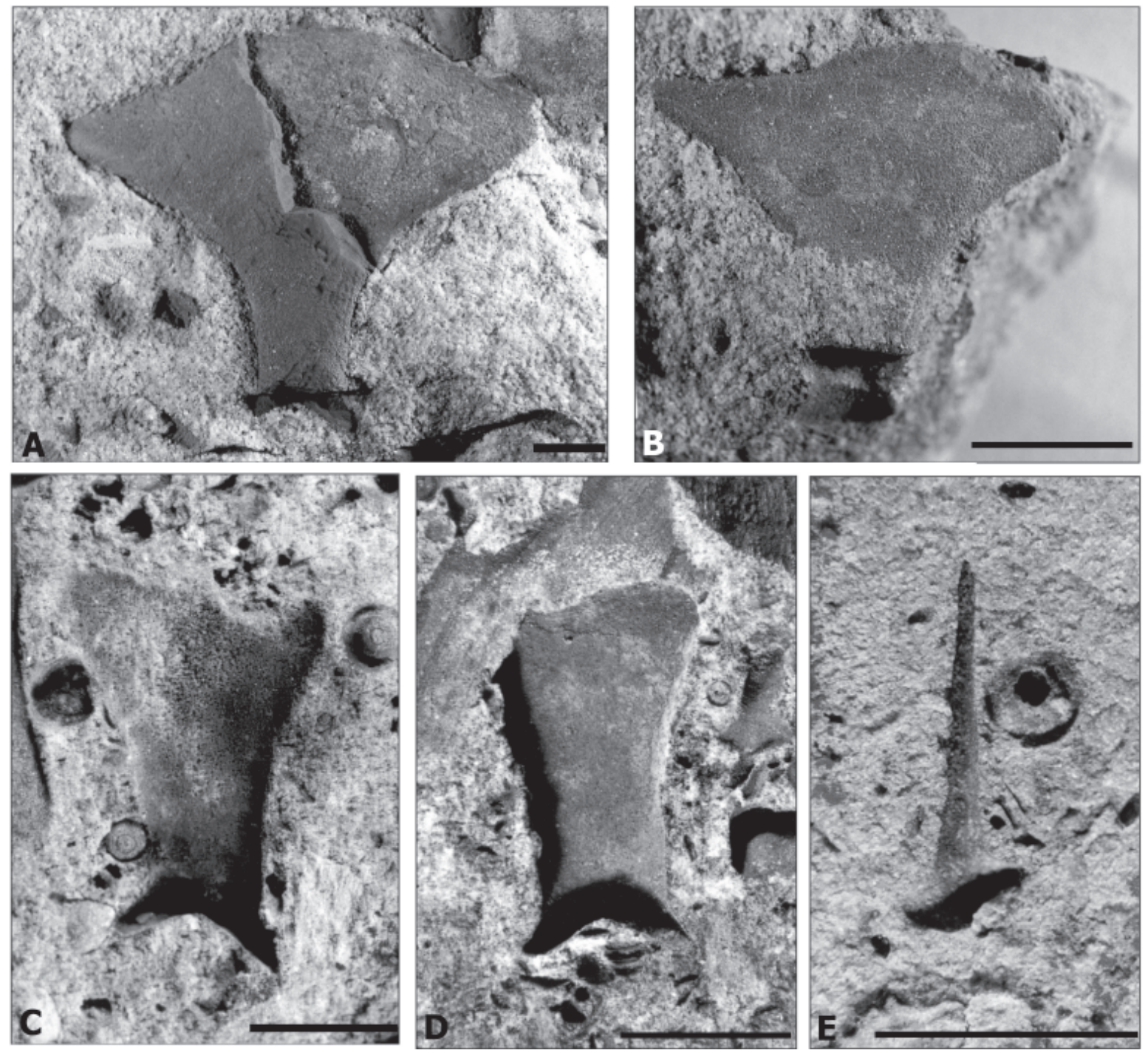

Figura 3. Placas calicinais de Monstrocrinus securifer. Placas calicinais com expansão em forma de machado: A, MN 5164; B, MN6133la; C, MN 5173a3; D, MN 5173a4; E, placa calicinal com expansão em forma de espinho em MN 5173a5. Escalas = $10 \mathrm{~mm}$.

Figure 3. Monstrocrinus securifer. Calicinal plates with ax-like expansions: A, MN 5164; B, MN 6133-la; C, MN 5173a3; D, MN 5173a4; E, calicinal plate with spine-like expansion em MN 5173a5. Scale bars $=10 \mathrm{~mm}$.

estão presentes placas calicinais com os dois tipos de expansões (uma robusta em forma de machado e outra em forma de espinho longo e estreito) referidas por Schmidt (1941) à M. securifer. Essas últimas diferem das placas calicinais de M. granosus por não possuírem a ornamentação granulosa, e pela forma de suas expansões que em $M$. granosus são estreitas, mas curvas e formadas por dois processos. Diferem também de Monstrocrinus sp. (Le Menn, 1990), por não apresentarem as pequenas estruturas arredondadas radiais localizadas em torno da base da expansão da placa calicinal, e porque as expansões incompletas das placas calicinais de Monstrocrinus sp., embora sejam finas, parecem se alargar distalmente ao contrário das expansões em forma de espinho dos exemplares brasileiros que se afinam em direção a sua extremidade distal. As placas calicinais portadoras de grandes expansões da bacia do Amazonas diferem das de $M$. aliformes pelo contorno em forma de machado e pela ausência das apófises ou espinhos curtos, presentes na espécie espanhola.
Subclasse e Ordem incertas [Grupo Cyclici]

Família ø FLUCTICHARACIDAE Moore \& Jeffords, 1968 $ø$ Laudonomphalus Moore \& Jeffords, 1968

$\varnothing$ Laudonomphalus regularis Moore \& Jeffords, 1968 (Figura 4, Tabela 1)

Material. MN 5173a6, MN 5173a7, MN 5173b1, MN 5173b2, MN 5173b8, MN 5173c1, MN 6133-Ic, MN 7418-Ib, MN 7581Ia, MN 7581-Id, MN 7582-Ia, MN 7583-I, MN 7584-Ia, MN 7587-I, MN 8196-Ia1 e MN 8196-Ib.

Descrição. Pedúnculo heteromórfico circular; noditaxis com quatro colunais; colunais nodais com latera (lados) assimétricas longitudinalmente e epifacetas em forma de quilha abaixo da meia altura da colunal; internodais com latera convexas longitidunalmente, simétricas a levemente assimétricas. Linha de sutura do tipo simplectial, crenulada. Articulum (faceta articular) grande contendo em torno de 50 


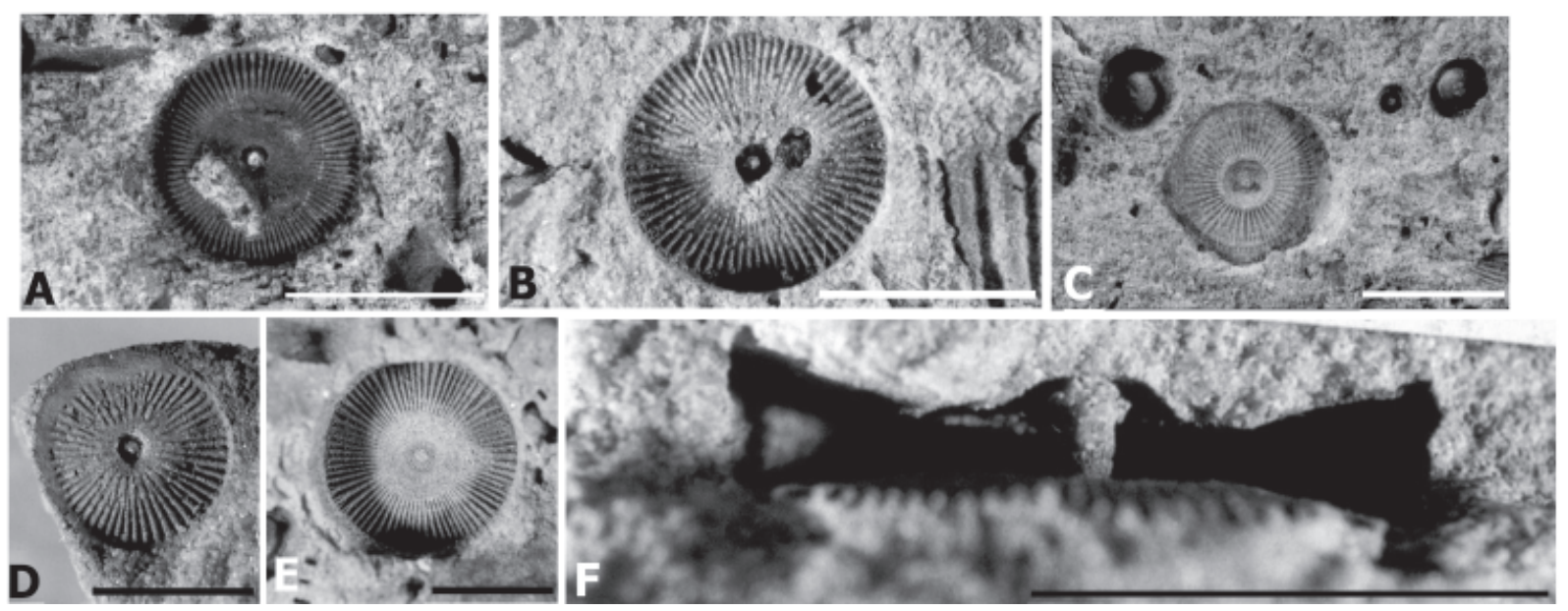

Figura 4. Laudonomphalus regularis. Colunais isoladas apresentando a morfologia da faceta articular e da latera lisa: A, MN 5173a6; B, MN 5173b8; C, MN 5173a7; D, MN 6133-Ic; E, MN 3412-I. F, MN 7581-Id, seção transversal de uma colunal isolada. Escalas = 10 mm. Figure 4. Laudonomphalus regularis. Isolated columnals showing the articular facet and the smooth latera morphology: A, MN 5173a6; B, MN 5173b8; C, MN 5173a7; D, MN 6133-Ic; E, MN 3412-I. F, cross section of an isolated columnal in MN 7581 -Id. Scale bars $=10 \mathrm{~mm}$.

culmina (cristas) médias a grossas, retas e longas, que podem ou não se ramificar próximo à margem da faceta (a grande maioria não se ramifica), aumentando em altura e diâmetro do centro para periferia; faceta articular ligeiramente côncava, com perilumen amplo, bem elevado e com dentículos grossos no topo; lumen circular, bastante diminuto.

Discussão. Moore \& Jeffords (1968) descreveram duas espécies de Laudonomphalus, L. ornatus e L. regularis, do Eriano dos Estados Unidos, cuja morfologia e índices das facetas articulares são semelhantes. A distinção entre elas está na morfologia externa das latera, lisas em L. regularis, e mais simétricas longitudinalmente e com tubérculos bem evidentes em L. ornatus. Com base na observação da morfologia das colunais dos espécimes da Formação Maecuru e das medidas de suas feições, concluiu-se que nesta formação ocorrem as duas espécies. Os espécimens referidos à $L$. regularis diferem dos norte-americanos pelo maior diâmetro das colunais e pelo perilumen mais amplo. Os espécimes da bacia do Amazonas diferem de L. tuberosus Moore \& Jeffords 1968, do Couviniano siberiano, por não possuírem tubérculos grandes nas latera e por apresentarem noditaxis de quatro colunais e não de duas colunais. Diferem também de L. seillouensis Le Menn, 1981, por possuírem pedúnculo heteromórfico, lumen circular em vez de pentagonal e colunais com maior diâmetro; de L. conicus (Le Menn, 1970), por possuírem faceta articular menos côncava, com crenulae mais finas e em maior número, lumen circular e não pentagonal e de maior diâmetro; de L. minimus (Le Menn, 1970) por possuírem pedúnculo heteromórfico, noditaxis com quatro colunais e não duas, colunais bicôncavas em vez de planas e com maior diâmetro; faceta articular com lumen circular e não pentagonal, perilumen mais evidente, maior número de crenulae, e por não possuírem a depressão anelar na periferia da faceta articular característica de L. minimus; de L. celticus (Le Menn, 1970), principalmente por características da faceta articular como lumen circular e não pentagonal, perilumen mais desenvolvido e por apresentar maior número de crenulae que se alargam do perilumen até a periferia da faceta articular, diferentemente de L. celticus onde as crenulae permanecem com tamanho constante; além disso, L. celticus possui menor diâmetro e apresenta, na margem externa da faceta articular, pequenas crenulae, que se intercalam entre as maiores originadas no perilumen, ausentes no material aqui estudado. O material da Formação Maecuru distingui-se de L. tenuicrenulatus por possuir colunal de maior diâmetro, lumen circular e não subpentagonal, perilumen mais desenvolvido e crenulae mais numerosas que, às vezes, se bifurcam próximo à margem externa da superfície articular. Difere de L. maximus (Le Menn, 1976) do Emsiano da França, Espanha e Bélgica por possuir noditaxis sempre com quatro colunais, enquanto em $L$. maximus o número de colunais no noditaxis pode variar de duas a oito, faceta articular menos côncava com lumen circular e não pentagonal e perilumen mais desenvolvido. Já $L$. marettensis Le Menn, 1981, da França e Espanha, difere do material da bacia do Amazonas por possuir vários tubérculos nas latera que muitas vezes se prolongam em espinhos e pelo menor número de crenulae, mais largas na faceta articular. Em relação à L. rotundilatus Le Menn, 1988, do Frasniano da

Tabela 1. Medidas (mm) e índices de L. regularis.

Table 1. Measurements $(\mathrm{mm})$ and index of $L$. regularis.

\begin{tabular}{|c|c|c|c|c|c|c|c|c|c|c|}
\hline & $\mid \begin{array}{c}\text { KD } \\
\text { nodal }\end{array}$ & $F$ & L & Li & $\mathbf{P}$ & $\mathbf{P i}$ & $\begin{array}{c}\mathrm{KH} \\
\text { nodal }\end{array}$ & $\begin{array}{c}\mathrm{KHi} \\
\text { nodal }\end{array}$ & NT & $\mathbf{N i}$ \\
\hline $\begin{array}{c}\text { MN } \\
7584-l a\end{array}$ & - & 10 & 0,5 & 5 & 4,3 & 43 & & & & \\
\hline $\begin{array}{c}\text { MN } \\
7581-l a\end{array}$ & - & 8 & 0,5 & 6,25 & 2,5 & 31,25 & & & & \\
\hline $\begin{array}{c}\text { MN } \\
7582-l a\end{array}$ & 6,1 & 5,6 & 0,5 & 8,9 & 1,5 & 26,8 & 0,9 & 14,8 & 2,3 & 39,1 \\
\hline $\begin{array}{c}\text { MN } \\
7583-I\end{array}$ & & 9,8 & 0,5 & 5,1 & 2,1 & 21,4 & & & & \\
\hline $\begin{array}{c}\text { MN } \\
5173 \mathrm{c1} 1\end{array}$ & & 12,5 & 0,7 & 5,6 & 3,1 & 24,8 & & & & \\
\hline $\begin{array}{c}M N \\
5173 b 1\end{array}$ & & 11,5 & 0,5 & 4,35 & 1,6 & 13,9 & & & & \\
\hline $\begin{array}{c}\text { MN } \\
5173 b 2\end{array}$ & & 12,2 & 0,7 & 5,7 & 3,1 & 25,4 & & & & \\
\hline
\end{tabular}




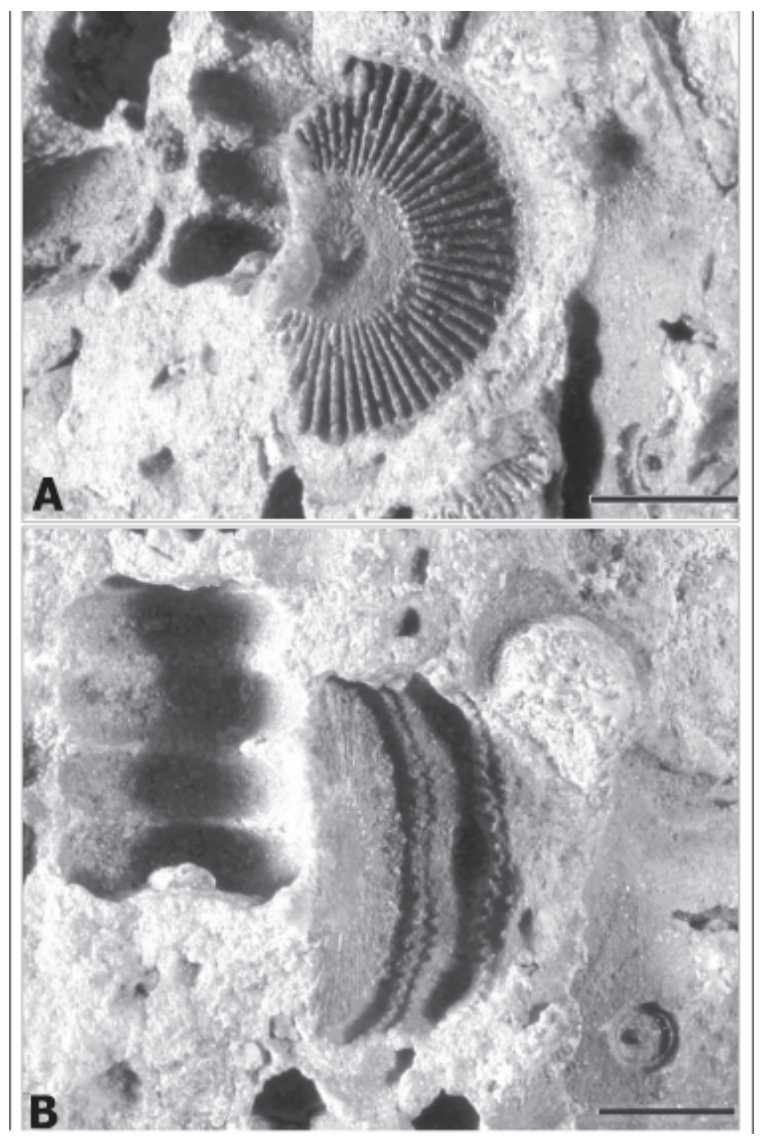

Figura 5. Laudonomphalus ornatus. MN 5173c2, pluricolunal apresentando a morfologia do articulum (A) e das latera com tubérculos (B), escalas $=5 \mathrm{~mm}$.

Figure 5. Laudonomphalus ornatus. MN 5173c2, pluricolumnal showing articulum morphology (A) and latera with tubercles (B), scale bars $=5 \mathrm{~mm}$.

França, o material aqui estudado difere por apresentar um padrão heteromórfico menos evidente com duas e não quatro ordens de internodais; em L. rotundilatus a tertinternodal é plano-convexa enquanto que no material da Formação Maecuru todas colunais são bicôncavas; L. rotundilatus também apresenta latera simétricas e o lumen, em algumas colunais, pode estar situado numa posição excêntrica.

\section{ø Laudonomphalus ornatus Moore \& Jeffords, 1968}

(Figura 5a,b; Tabela 2)

Material. MN 5173b3, MN 5173c2 e MN 5173c3.

Descrição. Pedúnculo heteromórfico circular, noditaxis com quatro colunais; colunais com latera simétricas longitudinalmente; nodais apresentando de seis a 10 tubérculos pequenos, bem evidentes nas latera; internodais com menor diâmetro e altura que as nodais, apresentando latera simétricas, lisas e levemente convexas longitudinalmente; linha de sutura do tipo simplectial, crenulada; articulum grande apresentando em torno de 50 cúlmens com crenulae médias a grossas, retas e longas, as quais podem ou não se ramificar, aumentando em altura e diâmetro do centro para a periferia; faceta articular ligeiramente côncava, com perilumen amplo, bem elevado e com dentículos grossos no topo; lumen circular, bastante diminuto.

Discussão. A maioria das diferenças encontradas entre $L$. regularis e as demais espécies do gênero podem ser estendidas para o material aqui identificado como L. ornatus, pois essas duas espécies apresentam características da faceta articular praticamente idênticas, diferindo apenas na presença de tubérculos nas latera, mais simétricas, de L. ornatus. Além de diferenças na faceta articular, os tubérculos nas latera de L. ornatus diferem dos tubérculos de L. tuberosos por serem menores e simétricos, e daqueles de $L$. marettensis e $L$. rotundilatus por serem menores e nunca se prolongarem em apófises ou espinhos. Segundo Le Menn (1988), no material coletado na região de Boulonnais, L. ornatus e L. regularis coexistem, assim como todos os estágios intermediários entre as latera com superfície lisa e com tubérculos bem desenvolvidos. Assim sendo, para Le Menn (1988), L. ornatus e $L$. regularis seriam variações ontogenéticas de uma mesma espécie, L. gr. regularis-ornatus. L. regularis corresponderia às colunais mais novas da região proximal e $L$. ornatus às colunais mais antigas da região distal do pedúnculo. $\mathrm{Na}$ Formação Maecuru as duas espécies também coexistem; porém, como não foram encontradas articuladas em um mesmo pedúnculo e como não ocorrem os estágios intermediários, optou-se por mantê-las como espécies separadas. O material da Formação Maecuru é mais semelhante ao material dos Estados Unidos, apesar de diferir deste último pelo maior diâmetro das colunais e pelo perilumen mais amplo.

Família ø EXAESIODISCIDAE Moore \& Jeffords, 1968 $ø$ Exaesiodiscus Moore \& Jeffords, 1968

Espécie-tipo. ø Exaesiodiscus acutus Moore \& Jeffords, 1968.

$$
\begin{gathered}
ø \text { Exaesiodiscus aff. minutus Moore \& Jeffords, } 1968 \\
\text { (Figura 6A-C; Tabela 3) }
\end{gathered}
$$

Material. MN 5173a1, MN 5173a2, MN 5173b4, MN 5173b5, MN 5173b6, MN 5173c4, MN 5173c5, MN 6133-Ib, MN 7581Ib, MN 7581-Ic, MN 7582-Ib, MN 7584-Ib, MN 7585-I, MN 7586-Ia, MN 7586-Ib, MN 8196-Ia2 e MN 8197-Ib.

Descrição. Pedúnculo heteromórfico, circular; noditaxis com quatro a oito colunais; nodais bem mais largas e altas que as internodais, exceto quando o noditaxis possui oito colunais, pois a priminternodal se aproxima em dimensões e forma das nodais; sutura provavelmente lisa; nodais sem cirros com grande epifaceta lisa, bastante convexa e assimétrica longitudinalmente; latera das internodais lisas e retas longitudinalmente; faceta articular da nodal côncava e bem deprimida, para recepção das internodais; crenularium com crenulae finas, curtas e simples; aréola lisa e lumen quinqüelobado de tamanho médio.

Tabela 2. Medidas ( $\mathrm{mm}$ ) e índices de L. ornatus.

Table 2. Measurements $(\mathrm{mm})$ and index of L.ornatus.

\begin{tabular}{c|cccccccccc} 
& $\begin{array}{c}\text { KD } \\
\text { nodal }\end{array}$ & $\mathbf{F}$ & $\mathbf{L}$ & $\mathbf{L i}$ & $\mathbf{P}$ & $\mathbf{P i}$ & $\begin{array}{c}\mathbf{K H} \\
\text { nodal nodal }\end{array}$ & $\mathbf{K h i}$ & $\mathbf{N i}$ \\
\hline $\begin{array}{c}\mathbf{M N} \\
\mathbf{5 1 7 3 c 2}\end{array}$ & 13,9 & 13,4 & 0,7 & 5,2 & 4,1 & 30,6 & 2,8 & 20,1 & 6,2 & 45,1 \\
$\mathbf{M N}$ & & 15,8 & 0,8 & 5,1 & 4,6 & 29,1 & & & & \\
$\mathbf{5 1 7 3 c 3}$ & & & & & & & & & & \\
$\begin{array}{c}\mathbf{M N} \\
\mathbf{5 1 7 3 b 3} 3\end{array}$ & 13,3 & 0,7 & 5,3 & 4,1 & 30,8 & & & & \\
\hline
\end{tabular}


Tabela 3. Medidas ( $\mathrm{mm}$ ) e índices de Exaesiodiscus aff. minutus. Entre parênteses o número de colunais do noditaxis.

Table 3. Measurements $(\mathrm{mm})$ and index of the Exaesiodiscus aff minutus. In parenthesis the number of noditaxis columnals.

\begin{tabular}{|c|c|c|c|c|c|c|c|c|c|c|c|}
\hline & $\begin{array}{l}\text { KD } \\
\text { nod }\end{array}$ & $F$ & $\mathbf{F i}$ & $\mathbf{L}$ & Li & A & $\mathbf{A} \mathbf{i}$ & $\begin{array}{l}\text { KH } \\
\text { nod }\end{array}$ & $\begin{array}{l}\mathrm{KHi} \\
\text { nod }\end{array}$ & NT & $\mathbf{N i}$ \\
\hline $\begin{array}{c}\text { MN } \\
5173 a\end{array}$ & 9 & 5,3 & 58,8 & & & & & 3,6 & 40 & $\begin{array}{l}5,9 \\
(4)\end{array}$ & 55,9 \\
\hline $\begin{array}{r}\text { MN } \\
5173 a\end{array}$ & 10 & 4 & 40 & 1,1 & 27,2 & 1,5 & 37,5 & & & & \\
\hline $\begin{array}{r}\text { MiN } \\
5173 \mathrm{c}\end{array}$ & 9,1 & 5,1 & 56,1 & 1,1 & 21,6 & 2,8 & 54,9 & 3,7 & 40.6 & & \\
\hline $\begin{array}{r}\text { MN } \\
5173 \mathrm{c}\end{array}$ & 7,6 & 4,9 & 64,5 & 1,2 & 24,5 & 1,9 & 38,8 & 2,9 & 38,2 & & \\
\hline $\begin{array}{c}\text { MN } \\
5173 b\end{array}$ & 8,5 & 5,1 & 60 & 0,9 & 17,6 & 2,3 & 45,1 & 2,7 & 31,8 & $\begin{array}{l}7,5 \\
(8)\end{array}$ & 36 \\
\hline $\begin{array}{r}\text { MN } \\
5173 b\end{array}$ & 7,2 & 3,8 & 52,8 & 0,9 & 23,7 & 1,4 & 36,8 & & & & \\
\hline 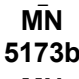 & 3,0 & 2,2 & 73,3 & & & & & 1,4 & 46,6 & $\begin{array}{l}4,2 \\
(8)\end{array}$ & 33,3 \\
\hline $\begin{array}{r}\text { MN } \\
7581-\end{array}$ & 5,3 & 3,8 & 71,7 & 0,9 & 23,7 & 2,1 & 55,6 & & & & \\
\hline $\begin{array}{r}\text { MN } \\
7581-\end{array}$ & 9,8 & 6,8 & 69,4 & 1,2 & 17,6 & 2,6 & 38,2 & & & & \\
\hline $\begin{array}{r}\text { MN } \\
7585-\end{array}$ & 6,7 & 4 & 59,7 & 1,5 & 37,5 & 1,4 & 35 & & & & \\
\hline $\begin{array}{r}\text { MN } \\
7586-\end{array}$ & 5,9 & 3,4 & 57,6 & & & & & 2,2 & 37,3 & $\begin{array}{c}3 \\
(4)\end{array}$ & 73,3 \\
\hline $\begin{array}{r}\text { MN } \\
7586-\end{array}$ & 8,7 & 4,4 & 50,6 & 0,8 & 18,2 & 2,5 & 56,8 & & & & \\
\hline
\end{tabular}

Discussão. As colunais do gênero Exaesiodiscus, citadas pela primeira vez para a Formação Maecuru por Ferreira \& Fernandes (1985), foram aqui identificadas como Exaesiodiscus aff. minutus por apresentar a epifaceta da nodal muito convexa e arredondada, espessando-se levemente em direção à periferia. Entretanto, diferem de $E$. minutus pelo maior diâmetro das colunais nodais, pela maior altura das nodais em relação ao diâmetro, pelo crenularium mais expandido e pelo lumen quinqüelobado diferente do lumen circular que ocorre no material da América do Norte (veja Moore \& Jeffords, 1968). Distingue-se de E. acutus por possuir maior faceta articular, internodais de maior diâmetro e latera convexas, e não anguladas; distingue-se de $E$. truncatus por possuir a epifaceta mais convexa, e pela faceta articular mais deprimida para recepção das internodais. Já $E$. salviensis difere do material aqui analisado por apresentar superfície articular mais côncava e aréola lisa (Le Menn \& Rachebouef,1976), diferente da superfície articular plana à ligeiramente côncava e aréola granulada do material brasileiro. Além disso, E. salviensis apresenta espinhos e cirros nas latera das nodais, ausentes nas formas da Formação Maecuru.

\section{CONSIDERAÇÕES PALEOGEOGRÁFICAS}

O mapa de Scotese \& McKerrow (1990) para o Devoniano Médio apresenta uma massa continental equatorial a tropical, a Laurússia ou Euroamérica, separada pelo oceano Réico do Gondwana, situado em latitudes subtropical a polar.

Nas reconstruções de Robardet et al. (1993), a Europa meridional (península Ibérica e a maior parte da França até o maciço Armoricano) e todas as regiões ao leste que constituem as zonas saxo-turingiana e moldanubiana até a Boêmia,

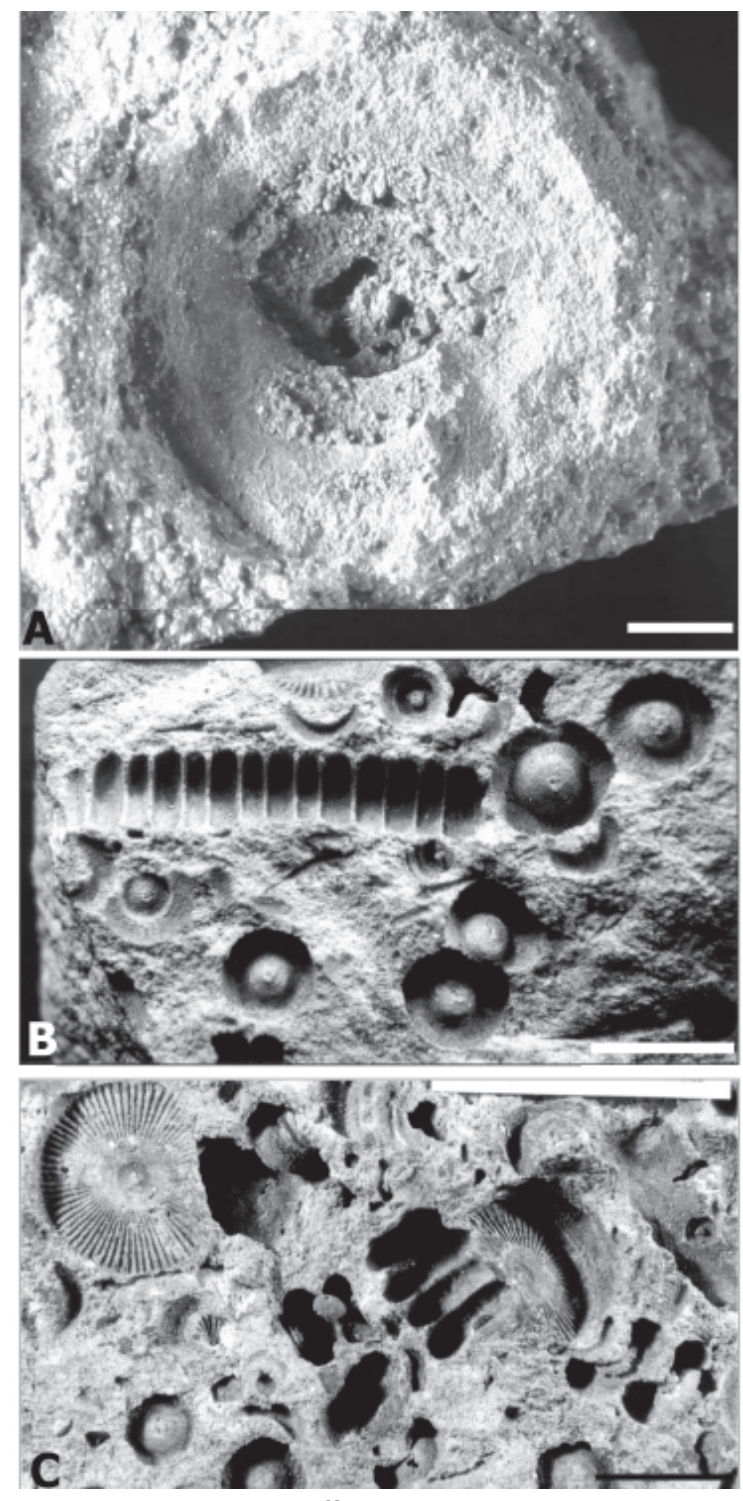

Figura 6. Exasiodiscus aff. minutus. A, MN 8197-lb, colunal apresentando o lumen pentalobado, escala $=2 \mathrm{~mm}$; B-C, vista geral dos pluricolunais e colunais com morfologia do articulum e do noditaxis, em MN7586-I (B) e MN 5173c (C), escalas $=10 \mathrm{~mm}$. Figure 6. Exasiodiscus aff. minutus. A, MN 8197lb, columnal with pentalobate lumen, scale bar $=2 \mathrm{~mm}$; B-C, general view of the columnals and pluricolumnals with articulum and noditaxis morphology, in MN7586-I (B) and MN 5173c (C), scale bars $=10 \mathrm{~mm}$.

formavam a Europa norte-gonduânica ou Armórica. Esta, no Paleozóico inferior (incluindo o Devoniano), estaria situada na borda norte da parte africana do Gondwana.Um dos principais problemas da paleogeografia do Devoniano é a relação entre as regiões norte-gonduânicas da Europa Central e Meridional (Armórica) e o cráton gonduânico. A maioria dos modelos baseados em paleomagnetismo propõe uma separação entre esses dois conjuntos, resultando na existência de um oceano Protétis. Entretanto, os dados sedimentares e paleofaunísticos não se coadunam com a presença de uma barreira de tal magnitude (Robardet et al.,1993; Scotese \& McKerrow, 1990), mas sim, a existência de espaços oceânicos estreitos, que não teriam alterado consideravelmente a unidade sedimentar e paleofaunística do conjunto. A ocorrência do gênero Monstrocrinus na bacia 
do Amazonas é mais um dado a favor da hipótese de um possível contato das comunidades marinhas da Armórica com as do norte do Gondwana.

De acordo com Le Menn (1990), o gênero Monstrocrinus é considerado raro no Devoniano e restrito ao Devoniano Inferior (topo do Emsiano). Suas espécies foram registradas na Alemanha (Maciço Xistoso Renano), na Espanha (região de Aragão) e na Argélia (montes de Ougarta). Esta ocorrência é de especial importância pelos seguintes motivos: (i) a forma da Formação Maecuru é idêntica à espécie-tipo do gênero, Monstrocrinus securifer, do Maciço Xistoso Renano, fato que sugere relação com a fauna bentônica desta região; (ii) é o primeiro registro do gênero na América do Sul; e (iii) é o primeiro registro do gênero no Devoniano Médio (Eifeliano). A presença de um táxon nas bacias brasileiras de idades mais recentes do que suas ocorrências em outros continentes não é um fato isolado; padrão similar é apresentado por alguns braquiópodes, entre eles Tropidoleptus carinatus (Conrad, 1841). Estes fatos fornecem subsídios importantes para interpretações paleogeográficas relacionadas ao padrão de distribuição e prováveis rotas de migração de invertebrados bentônicos no norte do Gondwana, durante o Devoniano.

\section{CONCLUSÕES}

Restos dissociados de crinóides encontrados na Formação Maecuru são aqui atribuídos à Monstrocrinus securifer, Laudonomphalus regularis, Laudonomphalus ornatus e Exaesiodiscus aff. minutus. A presença de Monstrocrinus no Devoniano Médio (Eifeliano) da bacia do Amazonas sugere uma possível rota de migração de Monstrocrinus, aparentemente originada no sudoeste da Europa no Devoniano Inferior, através do norte do Gondwana Oriental, atingindo a bacia do Amazonas no Devoniano Médio. Reforça, também, a hipótese de um possível contato entre as faunas marinhas bentônicas do norte do Gondwana e a Armórica.

\section{REFERÊNCIAS}

Bronn, H.G. 1840. Ctenocrinus ein neues Krinoiden-Geschlecht der Grauwacke. Neues Jahrbuch für Mineralogie, Geologie und Paläontologie, p. 542-548.

Conrad, T.A. 1841. Fifth annual report on the paleontology of the State of New York. New York Geological Survey, Annual Report 5:25-67.

Cunha, P.R.C.; Gonzaga, F.G.; Coutinho, L.F.C. \& Feijó, F.J. 1994. Bacia do Amazonas. Boletim de Geociências da Petrobrás, 8:47-55.

Derby, O.A. 1878. Contribuições para a geologia da região do baixo Amazonas. Archivos do Museu Nacional, 2:77-107.

Donovan, S.K. 2001. Nomenclature of disarticulated Pelmatozoan columnals:a comment. Journal of Palaeontology, 75:888-889.

Ferreira, C.S. \& Fernandes, A.C.S. 1985. Notas sobre alguns crinóides do Devoniano da Amazônia. Anais da Academia Brasileira de Ciências, 57(1):139.

Ferreira, C.S. \& Fernandes, A.C.S. 1989. Crinóides do Devoniano do Brasil. In: CONGRESSO BRASILEIRO DE PALEONTOLOGIA, 11, 1989. Resumo das Comunicações, Curitiba, SBP, p.23-24.

Fernandes, A.C.S.; Fonseca, V.M.M. \& Ferreira, C.S.F. 2000. Occurrence of Monstrocrinus securifer Schmidt, 1941 (Crinoidea
- Camerata) in the Middle Devonian of Amazon Basin, State of Pará, Brasil. In: INTERNATIONAL GEOLOGICAL CONGRESS, 31, 2000. Abstracts, Rio de Janeiro, CD-ROM.

Katzer, F. 1903. Grundzüge der Geologie des unteren Amazonasgebeietes (des Staates Pará in Brasilien). Leipzig, Max Weg., 296 p.

Katzer, F. 1933. Geologia do Estado do Pará (Brasil) . Boletim do Museu Paraense Emílio Goeldi de História Natural e Etnografia, 9:1-269.

Le Menn, J. 1970. Les crinoïdes du Sieginien supérieur da la rade de Brest (Finistère). Faculté des Sciences de l'Université de Rennes, These de Docteur, $107 \mathrm{p}$.

Le Menn, J. 1981. Les crinoïdes. Mémoires de la Societé Géologique et Minéralogique deBretagne, 24:261-273.

Le Menn, J. 1988. Equinodermes du Givetien et du Frasnien du Boulonnais (France). In: D. Brice (ed.) Le Dévonien de Ferques, Bas-Boulonnais (N. France). Université de Bretagne Occidentale, Collection Biostratigraphie du Paleozoïque, 7:455-477.

Le Menn, J. 1990. Présence de Monstrocrinus (Crinoidea) dans 1' Emsian Supérieur du domaine Nord-Gondwanien (Espagne, Algerie). Annales de Paléontologie, 76:149-158.

Le Menn, J. \& Rachebouef, P.R. 1976. Brachiopodes Chonetacea et crinoides des formatioms du Faou et de Montguyon (Dévonien Inferieur du Massif Armoricain). Annales de Societé Géologique du Nord, 96:283-323.

Loboziak, S. \& Melo, J.H.G. 2000. Miospores events from late Early to Late Devonian strata of Western Gondwana. Geobios, 33(4):399-407.

Loboziak, S. \& Melo, J.H.G. 2002. Devonian miospores succesions of Western Gondwana: update and correlation with Southern Euroamerican miospores zones. Review of Paleobotany and Palynology, 121:133-148.

Machado, D.M.C.; Fonseca, V.M.M. \& Rego, L.V.M. 1996. Estudos preliminares sobre a distribuição espacial da macrofauna da Formação Maecuru (Devoniano Médio) da bacia do Amazonas, Estado do Pará. In: SIMPÓSIO SUL-AMERICANO DO SILURO-DEVONIANO, 1, 1996. Anais, Ponta Grossa, Prefeitura de Ponta Grossa/UFPG/UFPR, p. 239-246.

Melo, J.H.G. 1988. The Malvinokaffric realm in the Devonian of Brazil. In: N.J. Mcmillan; A.F. Embry \& O.J. Glass (eds.) Devonian of the world, Canadian Society of Petroleum Geologists, p. 667-703.

Moore, R.C. \& Jeffords, R.M. 1968. Classification and nomenclature of fossil crinoids based on studies of dissociated parts of their columns. The University of Kansas Contributions, 9:1-86.

Moore, R.C.; Jeffords, R.M. \& Miller, T.H. 1968. Morphological features of crinoid columns. The University of Kansas Contributions, 8:1-30.

Robardet, M.; Blaise, J.; Bouyx, E.; Gourvennec, R.; Lardeaux, H.; Le Hérrissé, A.; Le Menn, J.; Melou, M.; Paris, F.; Plusquellec, et al. 1993. Paléogéographie de l'Europe occidentale de l'Ordovicien au Devonian. Bulletin de la Societé Géologique de France, 164:683-695.

Schmidt, W. 1941. Die Crinoïdeen des Rheinischen Devons. II. Die Crinoïdeen des Unterdevons bis zur Cultrijugaltus-Zone (mit Asschluss des Hunsrückschiefers). Abhandlungen der Reichsstelle für Bodenforschung, 182:1-253.

Scotese, C.R. \& Mckerrow, W.S. 1990. Revised world maps and introduction. In: W.S. Mckerrow \& C.R. Scotese (eds.) Palaeozoic palaeogeography and biogeography, The Geological Society of London, 21 p. (Memoir 12)

Received in August, 2005 ; accepted in May, 2006. 